\title{
Association of Small Dense Low-density Lipoprotein Cholesterol in Type 2 Diabetics with Coronary Artery Disease
}

\author{
Ya-Ching Huang ${ }^{1,2}$, Pi-Yueh Chang ${ }^{1,2}$, Jawl-Shan Hwang ${ }^{3}$, Hsiao-Chen Ning ${ }^{1,2,4}$
}

Background: The risk of coronary artery disease (CAD) increases two- to fourfold in diabetes. Small dense low-density lipoprotein (sdLDL) particles have been linked to an increased risk for CAD. In this study, we sought to compare the sdLDL cholesterol (sdLDL-C) level between the healthy control group and diabetics with CAD in the Taiwanese population.

Methods: $\quad$ Serum specimens were collected from healthy females and males of various age groups $(n=294)$, type 2 diabetics $(\mathrm{DM})$ without complications $(n=113)$, and patients having DM with CAD (DM-CAD) $(n=46)$. The commercial kit was used for the measurement of sdLDL-C level, which employs a simpler method. After heparin-magnesium precipitation of lipoproteins with density $<1.044 \mathrm{~g} / \mathrm{ml}$, sdLDL (density $=1.044-1.063 \mathrm{~g} / \mathrm{ml}$ ) remained in the supernatant and this sdLDL-C was measured using an automated chemistry analyzer.

Results: $\quad$ The sdLDL-C level was significantly higher in males than in females $(p<0.001)$ and there was an age effect on sdLDL-C $(p<0.001)$. The DM-CAD group had significantly higher sdLDL-C levels than the healthy control

\begin{abstract}
At a Glance Commentary
Scientific background of the subject

Small dense low-density lipoprotein (sdLDL) particles are associated with increased risk for coronary artery disease (CAD) and diabetes mellitus. We sought to compare the sdLDL cholesterol (sdLDL-C) level between the healthy control group and diabetics with CAD in the Taiwanese population.
\end{abstract}

\section{What this study adds to the field}

The DM-CAD group had significantly higher sdLDL-C levels than the healthy control group. There was an age effect on sdLDL-C in both males and females. The sdLDL-C level together with the LDL-C level are better risk assessment markers for type 2 diabetics with CAD than the LDL-C level alone. group $(p<0.001)$, but there was no statistical difference in the LDL-C level between DM-CAD group and the healthy control group. In addition, only individuals having both high LDL-C and sdLDL-C levels had a higher risk for DM-CAD, compared to those with low LDL-C levels and low sdLDL-C levels [Odds Ratio (OR) 4.97; 95\% Confidence Interval (CI) $1.96-12.57 ; p=0.001]$.

Conclusions: Our data suggest that the sdLDL-C level together with the LDL-C level are better risk assessment markers for type 2 diabetics with CAD than the LDL-C level alone.

(Biomed J 2014;37:375-379)

Key words: coronary artery disease, low-density lipoprotein cholesterol, small dense low-density lipoprotein cholesterol, Taiwanese population, type 2 diabetics

$\mathrm{L}$ ow-density lipoprotein cholesterol (LDL-C) has been considered as a critical risk factor in the development of coronary artery disease (CAD). The decision to treat
CAD is based on LDL-C levels. ${ }^{[1]}$ However, large CAD prevention trials have demonstrated that the reduction of LDL-C levels with lipid-lowering therapy dose not entirely

From the ${ }^{1}$ Department of Laboratory Medicine, Chang Gung Memorial Hospital at Linkou, Chang Gung University College of Medicine, Taoyuan, Taiwan; ${ }^{2}$ Department of Medical Biotechnology and Laboratory Science, College of Medicine, Chang Gung University, Taoyuan, Taiwan; ${ }^{3}$ Department of Metabolism and Endocrinology, Chang Gung Memorial Hospital at Linkou, Gung University College of Medicine, Taoyuan, Taiwan; ${ }^{4}$ Healthy Aging Research Center, Chang Guang University, Taoyuan, Taiwan Received: Mar. 13, 2013; Accepted: Dec. 30, 2013

Correspondence to: Hsiao-Chen Ning, Department of Laboratory Medicine, Chang Gung Memorial Hospital at Linkou. 5, Fusing St, Gueishan, Taoyuan 333, Taiwan (ROC). Tel: 886-3-3281200 ext. 2557; Fax: 886-3-3971827; E-mail: ning@ adm.cgmh.org.tw

DOI: $10.4103 / 2319-4170.132883$ 
eliminate coronary disease events. ${ }^{[2-6]}$ It has been suggested that small dense LDL (sdLDL) particles are highly atherogenic because they have a greater ability to penetrate the subendothelial layer, have longer residence times, and are oxidized more, compared to large, buoyant LDL. ${ }^{[7]}$ In several studies, sdLDL particles have been linked to an increased risk for CAD. ${ }^{[8-11]}$

Type 2 diabetes mellitus (DM), as a heterogeneous disease, along with hyperglycemia causes many acute and chronic complications including microvascular complications (nephropathy, neuropathy, and retinopathy) and macrovascular complications (CAD, stroke, and peripheral vascular disease). ${ }^{[2]}$ Type 2 diabetic patients have a two- to fourfold increased risk of CAD, compared to non-diabetic individuals. DM is associated with a cluster of interrelated plasma lipid and lipoprotein abnormalities, including reduced high-density lipoprotein (HDL) cholesterol, elevated triglycerides, and a predominance of sdLDL particles. ${ }^{[13]}$ However, these abnormalities occur in many patients despite them having a normal LDL-C level. ${ }^{[13]}$ There is evidence that each of these dyslipidemic features is associated with increased risk of cardiovascular disease, the leading cause of death in patients with DM. ${ }^{[14]}$ Taken together with the information described in the first paragraph, these findings suggest that sdLDL may have a predictive value for DM patients with CAD.

The sdLDL is traditionally measured by ultracentrifugation or gradient gel electrophoresis to measure the density or LDL particle size, respectively. ${ }^{[15,16]}$ Both methods are laborious and require special equipment and a long running time. Nuclear magnetic resonance (NMR) imaging is capable of simultaneously determining the size and number of LDL particles. ${ }^{[17]}$ However, the instrumentation and devices required for NMR are too costly for general clinical laboratories to acquire them. Hirano et al. have developed a method that uses heparin sodium salt precipitation followed by centrifugation for measuring sdLDL cholesterol (sdLDL-C). ${ }^{18]}$ Although sdLDL-C has been evaluated in the Japanese and US populations, it has not been studied in the Taiwanese population. In the current study, we report the measurement of sdLDL-C according to the method of Hirano et al. in the Taiwanese population, and the association of sdLDL-C levels in DM with CAD. ${ }^{[19,20]}$

\section{METHODS}

\section{Subjects}

Serum specimens, collected from healthy female and male individuals of various age groups (20-29, 30-39, 40-49, 50-59, and $\geq 60$ years old) visiting Chang Gung Memorial Hospital for an annual health checkup, were chosen as the first control group for the current study. All individuals selected for this study had a normal chemistry profile, including a normal lipid profile [triglycerides $(\mathrm{TG})<150 \mathrm{mg} / \mathrm{dl}$, total cholesterol (T-CHO) $<200 \mathrm{mg} / \mathrm{dl}$, HDL-C in male $\geq 40 \mathrm{mg} / \mathrm{dl}$, HDL-C in female $\geq 50 \mathrm{mg} / \mathrm{dl}$, LDL-C $<130 \mathrm{mg} / \mathrm{dl}$ ], normal fasting serum glucose levels ( $\leq 105 \mathrm{mg} / \mathrm{dl})$, and normal liver and kidney function tests. The numbers of female and male patients individuals were 149 and 145, respectively. In addition, 46 serum specimens of type 2 diabetic patients with coronary artery disease (DM-CAD group) (26 males and 20 females, mean age $65.5 \pm 8.7$ years, ranging from 50 to 82 years) were collected. DM-CAD group consisted of patients with acute myocardial infarction who were in the hospital during this study $(n=13)$, patients with previous myocardial infarction $(n=16)$, and patients with angina pectoris $(n=17)$. The diagnoses of myocardial infarction and angina pectoris were based on clinical symptoms, electrocardiography (ECG) changes, blood examinations, and coronary arteriograms. Besides, 113 serum specimens of type 2 diabetic patients without complications (64 males and 49 females, mean age $63.3 \pm 7.3$ years, ranging from 51 to 81 years) were collected. This group was gender- and age-matched with the DM-CAD group. These patients had no heart disease, blood vessel disease, neuropathy, nephropathy, or retinopathy. Moreover, 92 healthy individuals from the first control group, with similar gender and age characteristics as the disease groups, were chosen as the second control group (52 males and 40 females, mean age $62.7 \pm 9.1$ years, ranging from 50 to 86 years).

\section{Laboratory measurements}

Levels of glycosylated hemoglobin (HbA1c), TG, T-CHO, HDL-C, and LDL-C were measured by standard laboratory procedures. The sdLDL-C was measured using the commercial kit (sdLDL-C "SEIKEN"; Denka Seiken, Tokyo, Japan). The pretreatment solution ( $200 \mu \mathrm{l})$ containing heparin sodium salt and $\mathrm{MgCl}_{2}$ was added to the sample cup of the centrifugation tube for filtration. The serum sample $(200 \mu \mathrm{l})$ was then added to the same cup. The mixture was incubated for $10 \mathrm{~min}$ at $37^{\circ} \mathrm{C}$. After precipitation of lipoproteins with density $<1.044 \mathrm{~g} / \mathrm{ml}$ using heparin-magnesium, sdLDL (density $=1.044-1.063 \mathrm{~g} / \mathrm{ml}$ ) remained in the supernatant. The supernatant was passed through the filter after centrifuging at $5000 \times g$ for $1 \mathrm{~min}$. The sdLDL-C of the collected supernatant was measured using an automated chemistry analyzer (type 7600-210; Hitachi Ltd, Tokyo, Japan) based on the principle of colorimetry. Between-run precision was determined to be $6.1 \%$ and $5.4 \%$ at low control $(28.7 \pm 1.7 \mathrm{mg} / \mathrm{dl}$, $n=10)$ and at high control $(61.7 \pm 3.3 \mathrm{mg} / \mathrm{dl}, n=10)$, respectively. Within-run precision was determined to be $3.2 \%$ and $1.6 \%$ at low control $(27.2 \pm 0.9 \mathrm{mg} / \mathrm{dl}, n=10)$ and at high control $(58.3 \pm 0.9 \mathrm{mg} / \mathrm{dl}, n=10)$, respectively. Since the influence of drugs on the variables of interest is minimum during the stable drug treatment period (every 3 months) in 
DM or DM-CAD group, all measurements were performed during the stable drug treatment period.

\section{Statistical analysis}

Comparisons of mean values between the groups were made with Student's $t$ test. The difference in frequency data was determined by Chi-squared test. Multiple logistic regression analysis was used to assess the independent association between the risk factors and the incidence of DM-CAD. Differences with $p<0.05$ were considered to be statistically significant.

\section{RESULTS}

\section{sdLDL-C in the healthy control group}

We first compared the distribution of sdLDL-C among females and males [Figure 1A] and then separated the healthy female and male population into five age groups [Figure 1B] in order to determine whether there was an age or a gender difference. We found that sdLDL-C level was significantly higher in males than in females (males: $38 \pm 14 \mathrm{mg} / \mathrm{dl}$, $n=145$; females: $27 \pm 11 \mathrm{mg} / \mathrm{dl}, n=149 ; p<0.001)$. We also found that there was an age effect on sdLDL-C in both males and females. The sdLDL-C level was significantly higher in males above 50 years old than those below 50 years old ( $42 \pm 16$ vs. $35 \pm 13 \mathrm{mg} / \mathrm{dl} ; p<0.01)$. Females above 40 years old had a higher sdLDL-C level than those below 40 years old $(31 \pm 10$ vs. $22 \pm 9 \mathrm{mg} / \mathrm{dl} ; p<0.001)$.

\section{sdLDL-C and various parameters in the study population}

Table 1 shows the levels of sdLDL-C and other CAD risk factors in the three groups - the second control group, the group with type 2 diabetes without complications (DM), and the group with type 2 diabetes with CAD (DM-CAD). This control group was gender- and age-matched with the disease groups. The DM group had significantly higher TG, T-CHO, LDL-C, and sdLDL-C levels than the healthy control group $(p<0.001)$ and had significantly lower HDL-C level than the healthy control group $(p<0.001)$. The DM-CAD group also had significantly higher TG and sdLDL-C levels than the healthy control group $(p<0.001)$ and had significantly lower HDL-C level than the healthy control group $(p<0.001)$. However, the DM-CAD group did not have significantly higher T-CHO and LDL-C levels than the healthy control group.

\section{The contribution of sdLDL-C as a risk factor for DM-CAD}

To further evaluate the contribution of LDL-C and sdLDL-C as a risk factor for DM-CAD, multivariate lo-
Table 1: sdLDL-C and various parameters in the study population

\begin{tabular}{|c|c|c|c|}
\hline Variable & $\begin{array}{l}\text { Control } \\
(n=92)\end{array}$ & $\underset{(n=113)}{\mathrm{DM}}$ & $\begin{array}{c}\text { DM-CAD } \\
(n=46)\end{array}$ \\
\hline Male/female & $52 / 40$ & $64 / 49$ & $26 / 20$ \\
\hline Age, years & $62.7 \pm 9.1$ & $63.3 \pm 7.3$ & $65.5 \pm 8.7$ \\
\hline $\begin{array}{l}\text { Triglyceride, } \\
\mathrm{mg} / \mathrm{dl}\end{array}$ & $89 \pm 27$ & $143 \pm 62 *$ & $169 \pm 89^{*}$ \\
\hline $\begin{array}{l}\text { Total cholesterol, } \\
\mathrm{mg} / \mathrm{dl}\end{array}$ & $177 \pm 15$ & $193 \pm 33 *$ & $179 \pm 42^{\dagger}$ \\
\hline HDL-C, mg/dl & $58 \pm 12$ & $50 \pm 13^{*}$ & $40 \pm 11 * \$$ \\
\hline LDL-C, mg/dl & $102 \pm 14$ & $116 \pm 30 *$ & $107 \pm 34$ \\
\hline sdLDL-C, mg/dl & $39 \pm 14$ & $57 \pm 21 *$ & $54 \pm 27 *$ \\
\hline$\%$ sdLDL-C, $\%$ & $38 \pm 11$ & $49 \pm 15^{*}$ & $50 \pm 20 *$ \\
\hline
\end{tabular}

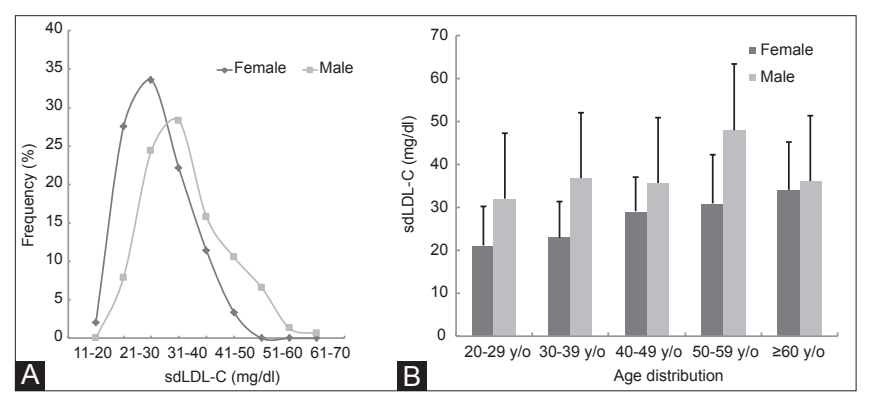

Figure 1: Normal values of sdLDL-C of both males and females and in various age groups: (A) The frequency distribution of sdLDL-C of healthy males $(n=145)$ and females $(n=149)$ with normolipidemia; (B) Normal sdLDL-C concentrations in various age groups and in males and females.

gistic regression analyses were performed. sdLDL-C level was significantly associated with DM-CAD after the adjustment for age and gender [Odds Ratio (OR) 1.05, 95\% Confidence Interval (CI) 1.02-1.07; $p<0.001]$. On the other hand, LDL-C alone was not a powerful marker associated with DM-CAD because no significant association between LDL-C and the risk for DM-CAD was found (OR 1.01, 95\% CI 0.99-1.03, $p=0.12$ ).

Figure 2 presents the synergy between LDL-C and sdLDL-C in the risk for DM-CAD after the adjustment for age and gender. Only individuals having both high LDL-C $>111 \mathrm{mg} / \mathrm{dl}$ (75\% percentile value of the second control group) and sdLDL-C $48 \mathrm{mg} / \mathrm{dl}$ (75\% percentile value of the second control group) were at an increased risk for DM-CAD (OR 4.97, 95\% CI 1.96-12.57; $p=0.001$ ). Interestingly, in individuals with high LDL-C levels in the absence of elevated sdLDL-C levels, the ORs were not calculated for DM-CAD because individuals with high LDL-C levels also had high sdLDL-C levels in the DM-CAD group. 


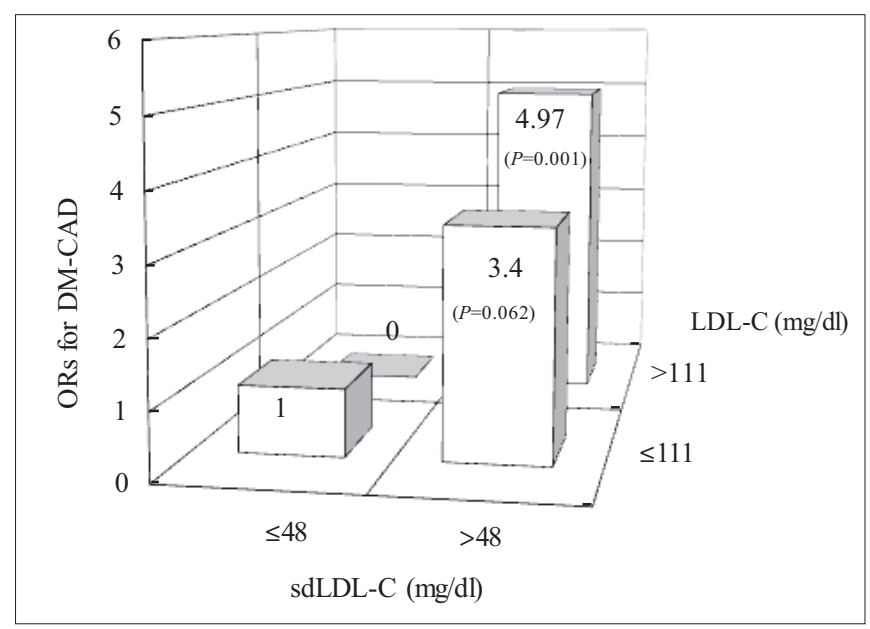

Figure 2: ORs for DM-CAD according to the LDL and sdLDL-C levels. The group with low LDL and sdLDL-C levels was used as a reference $(\mathrm{OR}=1.0)$. ORs were obtained by using logistic regression and were adjusted for age and gender.

\section{DISCUSSION}

In this study, the sdLDL-C level together with the LDL-C level, as a risk factor for DM patients with CAD, has been evaluated for the first time in the Taiwanese population by using a relatively simple method developed by Hirano et al. ${ }^{[18]}$ Our data suggest that sdLDL-C together with LDL-C shows a stronger association with CAD in patients with type 2 diabetes than LDL-C alone.

We observed that there are gender and age differences in sdLDL-C levels among our tested population, consistent with the data obtained in the Western populations. ${ }^{[19,20]}$ Although we have not separated menopausal and premenopausal women, women above 40 years old had a higher sdLDL-C concentration than those below 40 years old. Several studies have consistently shown more favorable lipoprotein profiles among premenopausal women than among men due to estrogen-related protective mechanisms. ${ }^{[21,22]} \mathrm{It}$ is well known that CAD risk markedly increases with aging in men and menopausal women, and alterations in LDL clearly contribute to this increased risk. ${ }^{[23]}$

In the present study, we also observed that patients with DM are associated with a cluster of interrelated plasma lipid and lipoprotein abnormalities. Such abnormalities include elevated TG, T-CHO, LDL-C, and sdLDL-C, as well as reduced HDL-C. Moreover, we observed that DM patients with CAD had no LDL-C elevation, but they had higher sdLDL-C levels than normolipidemic individuals, despite the use of cholesterol-lowering medication. Such findings may in part explain why statin treatment is of great benefit in CAD risk reduction associated with a reduction in LDL-C concentration, but very substantial residual CAD risk still remains. ${ }^{[24]}$ Rosuvastatin, the latest statin to be introduced in the market, seems to be more efficient in modulating plasma lipids and LDL subfractions. ${ }^{[25]}$

Although the level of LDL-C in our DM patients with CAD was not higher than that in our healthy controls, sdLDL-C and TG were substantially increased in DM patients with CAD. Since the sdLDL-C level is strongly associated with the serum TG level, an increase of sdLDL particles in CAD may be caused by an increase of serum TG.

In summary, our findings support the notion that the measurement of sdLDL-C concentration together with LDL-C concentration provides a more accurate assessment of the risk for DM with CAD than measuring LDL-C alone. However, more prospective studies are needed to determine whether sdLDL-C is an independent biomarker of DM with CAD. In addition, since the improved method using heparin sodium salt precipitation followed by centrifugation for measuring sdLDL-C is relatively simple and easier than other methods such as density gradient ultracentrifugation, gradient gel electrophoresis, or NMR, it is feasible to adopt such a method to measure sdLDL-C in general clinical laboratories.

\section{Acknowledgment}

We are grateful to the Resource Center for Clinical Research, Chang Gung Memorial Hospital for helpful data analysis.

\section{REFERENCES}

1. Carmena R, Duriez P, Fruchart JC. Atherogenic lipoprotein particles in atherosclerosis. Circulation 2004;109 (23 Suppl 1):III2-7.

2. Randomised trial of cholesterol lowering in 4444 patients with coronary heart disease: The Scandinavian Simvastatin Survival Study (4S). Lancet 1994;344:1383-9.

3. Shepherd J, Cobbe SM, Ford I, Isles CG, Lorimer AR, MacFarlane PW, et al. Prevention of coronary heart disease with pravastatin in men with hypercholesterolemia. West of Scotland Coronary Prevention Study Group. N Engl J Med 1995;333:1301-7.

4. MRC/BHF Heart Protection Study of cholesterol lowering with simvastatin in 20,536 high-risk individuals: A randomised placebo-controlled trial. Lancet 2002;360:7-22.

5. Sever PS, Dahlof B, Poulter NR, Wedel H, Beevers G, Caulfield M, et al. Prevention of coronary and stroke events with atorvastatin in hypertensive patients who have average or lower-than-average cholesterol concentrations, in the Anglo-Scandinavian Cardiac Outcomes Trial--Lipid Lowering Arm (ASCOT-LLA): A multicentre randomised controlled trial. Lancet 2003;361:1149-58.

6. Colhoun HM, Betteridge DJ, Durrington PN, Hitman GA, Neil HA, Livingstone SJ, et al. Primary prevention of cardiovascular disease with atorvastatin in type 2 diabetes in the Collaborative Atorvastatin Diabetes Study (CARDS): Multicentre randomised placebo-controlled trial. Lancet 2004;364:685-96.

7. Griffin BA. Lipoprotein atherogenicity: An overview of current mechanisms. Proc Nutr Soc 1999;58:163-9. 
8. Gardner CD, Fortmann SP, Krauss RM. Association of small low-density lipoprotein particles with the incidence of coronary artery disease in men and women. JAMA 1996;276:875-81.

9. St-Pierre AC, Cantin B, Dagenais GR, Mauriege P, Bernard PM, Despres JP, et al. Low-density lipoprotein subfractions and the long-term risk of ischemic heart disease in men: 13-year follow-up data from the Quebec Cardiovascular Study. Arterioscler Thromb Vasc Biol 2005;25:553-9.

10. Koba S, Yokota Y, Hirano T, Ito Y, Ban Y, Tsunoda F, et al. Small LDL-cholesterol is superior to LDL-cholesterol for determining severe coronary atherosclerosis. J Atheroscler Thromb 2008;15:250-60.

11. Hirayama S, Miida T. Small dense LDL: An emerging risk factor for cardiovascular disease. Clin Chim Acta 2012;414:215-24.

12. Bos M, Agyemang C. Prevalence and complications of diabetes mellitus in Northern Africa, a systematic review. BMC Public Health 2013;13:387-93.

13. Haffner SM. Management of dyslipidemia in adults with diabetes. Diabetes Care 2003;26 Suppl 1:S83-6.

14. Krauss RM. Lipids and lipoproteins in patients with type 2 diabetes. Diabetes Care 2004;27:1496-504.

15. Swinkels DW, Hak-Lemmers HL, Demacker PN. Single spin density gradient ultracentrifugation method for the detection and isolation of light and heavy low density lipoprotein subfractions. J Lipid Res 1987;28:1233-9.

16. Nichols AV, Krauss RM, Musliner TA. Nondenaturing polyacrylamide gradient gel electrophoresis. Methods Enzymol 1986;128:417-31.

17. Otvos JD, Jeyarajah EJ, Bennett DW, Krauss RM. Development of a proton nuclear magnetic resonance spectroscopic method for determining plasma lipoprotein concentrations and subspecies distributions from a single, rapid measurement. Clin Chem 1992;38:1632-8.

18. Hirano T, Ito Y, Koba S, Toyoda M, Ikejiri A, Saegusa H, et al. Clinical significance of small dense low-density lipoprotein cholesterol levels determined by the simple precipitation method. Arterioscler Thromb Vasc Biol 2004;24:558-63.

19. Ai M, Otokozawa S, Asztalos BF, Ito Y, Nakajima K, White CC, et al. Small dense LDL cholesterol and coronary heart disease: Results from the Framingham Offspring Study. Clin Chem 2010;56:967-76.

20. Vekic J, Zeljkovic A, Jelic-Ivanovic Z, Spasojevic-Kalimanovska V, Bogavac-Stanojevic N, Memon L, et al. Small, dense LDL cholesterol and apolipoprotein B: Relationship with serum lipids and LDL size. Atherosclerosis 2009;207:496-501.

21. Campos H, McNamara JR, Wilson PW, Ordovas JM, Schaefer EJ. Differences in low density lipoprotein subfractions and apolipoproteins in premenopausal and postmenopausal women. J Clin Endocrinol Metab 1988;67:30-5.

22. Chen CC, Huang TL. Association of serum lipid profiles with depressive and anxiety disorders in menopausal women. Chang Gung Med J 2006;29:325-30.

23. Executive summary of the third report of the National Cholesterol Education Program (NCEP) Expert Panel on detection, evaluation, and treatment of high blood cholesterol in adults (Adult Treatment Panel III). JAMA 2001;285:2486-97.

24. Cannon CP, Steinberg BA, Murphy SA, Mega JL, Braunwald E. Meta-analysis of cardiovascular outcomes trials comparing intensive versus moderate statin therapy. J Am Coll Cardiol 2006;48:438-45.

25. Rizzo M, Berneis K, Spinas GA, Rini GB, Kapur NK. Quantitative and qualitative effects of rosuvastatin on LDL-cholesterol: What is the clinical significance? Int J Clin Pract 2009;63:478-85. 